\title{
Immune checkpoint inhibitor associated myocarditis occurs in both high-grade and low-grade forms
}

\author{
Samantha N. Champion ${ }^{1} \cdot$ James R. Stone ${ }^{1}$ \\ Received: 7 June 2019 / Revised: 20 August 2019 / Accepted: 21 August 2019 / Published online: 18 September 2019 \\ (c) The Author(s), under exclusive licence to United States \& Canadian Academy of Pathology 2019
}

\begin{abstract}
Immune checkpoint inhibitor therapy for malignancy has been associated with adverse events including myocarditis. It has been unclear if there are distinct pathologic grades of this myocarditis that are associated with distinct clinical outcomes. Cardiac tissue from ten patients with immune checkpoint inhibitor myocarditis (nine biopsies and one autopsy) were evaluated using immunohistochemistry for CD3, CD8, CD68, tryptase, PD-L1, and C4D. The immune checkpoint inhibitor myocarditis cases were classified as either high grade $\left(>50 \mathrm{CD}^{+}\right.$cells/hpf) or low grade $\left(\leq 50 \mathrm{CD} 3^{+}\right.$cells/hpf). The densities of macrophages, $\mathrm{T}$ cells, eosinophils, necrotic myocytes, and PD-L1 ${ }^{+}$macrophages and myocytes were compared between the two groups and with 13 cases of grade $2 \mathrm{R}$ acute cellular allograft rejection. Three patients were classified as high-grade myocarditis and seven as low grade. There were higher densities of $\mathrm{CD}^{+}$cells and $\mathrm{CD} 8^{+}$cells in high-grade immune checkpoint inhibitor myocarditis and rejection compared with low-grade myocarditis. The number of $\mathrm{CD} 68^{+}$macrophages was higher in high-grade myocarditis compared with low-grade myocarditis and rejection. For both grades of myocarditis, there was a higher CD68/CD3 ratio and a higher density of PD-L1 ${ }^{+}$macrophages and myocytes compared with rejection. Clinically, there were trends toward higher serum troponin levels and shorter interval from first immune checkpoint inhibitor treatment in the high-grade myocarditis group compared with the low-grade group. All the patients with high-grade myocarditis died, while all the patients with low-grade myocarditis were still living. These data suggest that immune checkpoint inhibitor myocarditis occurs in two forms, a high-grade form with increased inflammatory cell infiltration and a more fulminant clinical course, and a low-grade form with a lower degree of inflammatory cell infiltration and a more indolent clinical course. Compared with acute cellular rejection, immune checkpoint inhibitor myocarditis is characterized by a more lymphohistiocytic inflammatory infiltrate with an increased CD68/CD3 ratio and increased $\mathrm{PD}-\mathrm{L} 1^{+}$macrophages and myocytes.
\end{abstract}

\section{Introduction}

Malignant cells may express neoantigens, and thus can be targeted by the immune system. However in some patients, the immune response is suppressed due to signaling through immune inhibitory receptors such as programmed cell death protein 1 (PD-1) and cytotoxic T-lymphocyte-associated protein 4 (CTLA-4) [1, 2]. This immune suppression may be facilitated by expression of the PD-1 ligand PD-L1 by the cancer cells. Blockade of these immune checkpoints can

James R. Stone

jrstone@mgh.harvard.edu

1 Department of Pathology, Massachusetts General Hospital, Harvard Medical School, Boston, MA 02114, USA be accomplished by the administration of specific antibodies, and such immune checkpoint inhibition has become an important new approach to treat malignancy [3]. However, immune checkpoint inhibition therapy can result in adverse reactions that mimic autoimmune processes. While often relatively benign, these immune-related adverse events affect multiple organ systems and occur in up to $70-90 \%$ of patients treated with immune checkpoint inhibitors [4-7].

One of the less common but more severe types of immune-related adverse events in the setting of immune checkpoint inhibition treatment is myocarditis [8]. Immune checkpoint inhibitor myocarditis only affects $\sim 1 \%$ of patients treated with immune checkpoint inhibitors but has a mortality of $\sim 50 \%$ [9-12]. Thus, gaining an understanding of the features of immune checkpoint inhibitor myocarditis that are associated with poor outcome is of much 
importance. Interestingly, there is significant variability in the clinical presentation of patients with immune checkpoint inhibitor myocarditis. While the median time to presentation from first immune checkpoint inhibitor treatment is typically on the order of 1-2 months, patients may present more than a year after initiating immune checkpoint inhibition therapy $[12,13]$. Likewise patients may experience fatal myocarditis after a single cycle of immune checkpoint inhibition treatment [14], or may not present with cardiotoxicity until after 30 infusions of immune checkpoint inhibition treatment [13]. It has been unclear as to whether or not there are specific pathologic features correlating with these varied clinical presentations.

Currently, reports of the pathologic features of immune checkpoint inhibitor myocarditis are limited to case reports and small case series [14-31]. These case reports have indicated the inflammatory infiltrate to be either lymphocytic or lymphohistiocytic. A small number of case reports have indicated the presence of eosinophils [20], and one case, which was published twice, was reported to also contain giant cells [20,32]. Immune checkpoint inhibitor myocarditis is often considered to be similar to cardiac allograft rejection, and this perceived similarity has been used as evidence for treating patients with immune checkpoint inhibitor myocarditis with medications that have been successful in the setting of cardiac allograft rejection, such anti-thymocyte globulin [11, 12, 14, 22]. However, it has been unclear how the pathology of immune checkpoint inhibitor myocarditis compares with more traditional myocardial inflammatory conditions such as cardiac allograft rejection. It has also been unclear if there are distinct pathologic grades of immune checkpoint inhibitor myocarditis and if such grades are associated with distinct clinical outcomes. Here we address these questions with a series of ten cases of immune checkpoint inhibitor myocarditis by classifying them into high-grade and low-grade groups, correlating the histologic grades with clinical presentation and outcome, and comparing the quantitative histologic features of immune checkpoint inhibitor myocarditis with those of cardiac allograft rejection.

\section{Materials and methods}

\section{Case selection}

The pathology database at Massachusetts General Hospital were searched to identify cases of myocarditis in the setting of immune checkpoint inhibition treatment from Jan 2016 up through Feb 2019. For each patient, only the initial cardiac pathology specimen, either endomyocardial biopsy or autopsy, was included in the primarily analysis [33]. As a comparison group, 13 consecutive endomyocardial biopsies of heart transplant patients with International Society of Heart and Lung Transplantation grade $2 \mathrm{R}$ acute cellular rejection obtained during 2017-2018 were also studied [34]. For each immune checkpoint inhibitor myocarditis case, the patient age, gender, left ventricular ejection fraction from echocardiography, serum troponin-T level, interval between first immune checkpoint inhibition treatment to biopsy/autopsy, number of cycles of immune checkpoint inhibition treatment, specific immune checkpoint inhibitor utilized, cancer type, and survival were obtained from the medical records. In a separate analysis, ten endomyocardial biopsies from cardiac transplant patients without rejection and ten native heart biopsies/resections without myocarditis were also utilized. The latter group consisted of seven native endomyocardial biopsies that were negative for myocarditis, as well as two resected left ventricular apical core segments and one surgically resected heart from patients with idiopathic dilated cardiomyopathy. The study was approved by the Hospital's human subjects institutional review board.

\section{Histologic staining and evaluation}

The cardiac pathology specimens were evaluated histologically by routine hematoxylin and eosin staining and by using immunohistochemistry for CD3, CD68, tryptase, and C4D as single stains and for CD8/PD-L1 as a double stain with CD8 in red and PD-L1 in brown [35]. All immunohistochemical stains were performed on routine automated clinical immunohistochemical staining devices. Cells staining positively were counted using an Olympus CX41 microscope with a $400 \times$ high power field (hpf) size of 0.85 $\mathrm{mm}^{2}$. The numbers of cells staining in ten (10) $400 \times \mathrm{hpfs}$ with the most inflammation were counted, and the data expressed as cells/hpf (mean \pm Standard Error (SE)). PD-L1 + macrophages and cardiac myocytes were each quantified based on the presence of diffuse membrane or diffuse cytoplasmic PD-L1 staining and on the morphology of the cells. Eosinophils were identified on the hematoxylin and eosin stained slides. The immune checkpoint inhibitor myocarditis cases were divided into two groups based on the density of $\mathrm{CD}^{+} \mathrm{T}$ cells: high-grade immune checkpoint inhibitor myocarditis $\left(>50 \mathrm{CD}^{+}\right.$cells/hpf) or low-grade immune checkpoint inhibitor myocarditis $\left(\leq 50 \mathrm{CD}^{+}\right.$cells/ hpf). The presence and/or densities of $\mathrm{CD}^{+}{ }^{+}$T-lymphocytes, $\mathrm{CD} 8^{+}$cytotoxic T-lymphocytes, $\mathrm{CD}^{+} 8^{+}$macrophages, tryptase ${ }^{+}$mast cells, eosinophils, necrotic $\mathrm{C}_{4} \mathrm{D}^{+}$ myocytes, and PD-L1 ${ }^{+}$macrophages and myocytes were compared between the three groups (high-grade immune checkpoint inhibitor myocarditis, low-grade immune checkpoint inhibitor myocarditis, grade $2 \mathrm{R}$ acute cellular rejection), as were the ratio of the $\mathrm{CD} 8^{+}$cells/CD3 ${ }^{+}$ cells $(\mathrm{CD} 68 / \mathrm{CD} 3)$, the ratio of the $\mathrm{CD} 8^{+}$cells/CD $3^{+}$cells 
(CD8/CD3) and the percentage of macrophages expressing PD-L1, which was calculated using the densities of the $\mathrm{PD}-\mathrm{L}^{+}{ }^{+}$macrophages and total $\mathrm{CD}^{+} 8^{+}$macrophages. The 20 heart biopsies and resections without myocarditis or rejection were evaluated by immunohistochemistry for PD-L1.

\section{Statistical analyses}

For the histologic assessments, since the data were skewed the continuous variables without values of zero were log transformed, and the three groups were compared with ANOVA with post-test by the Bonferoni method. Continuous variables, in which there were values of zero for any of the cases, were converted to dichotomous variables based on the presence or absence of the variable and/or an optimized threshold for the variable, and these dichotomous variables were compared using Fisher Exact test. Clinical features were compared between the two immune checkpoint inhibitor myocarditis groups using $t$-test, chi square test, or Fisher exact test as appropriate. Correlations were assessed by linear regression. $P$ values $<0.05$ were considered significant.

\section{Results}

Ten cases of immune checkpoint inhibitor myocarditis were identified that met the study criteria, nine cases of endomyocardial biopsy and one autopsy (Table 1). There were four patients with melanoma, two patients with pulmonary adenocarcinoma, and one patient each with renal cell carcinoma, cholangiocarcinoma, oral squamous cell carcinoma, and salivary gland mucoepidermoid carcinoma. All cases showed lymphocyte-rich inflammation on hematoxylin and eosin stain (Fig. 1). Eight of the ten cases met full Dallas criteria for myocarditis with clear myocyte injury. In all eight of these cases the myocyte injury was in discrete foci, and in no case was the infiltrate and injury diffuse throughout the specimen. In the autopsy case, discrete foci of myocarditis were present in every tissue block sampled from both ventricles. The other two cases were borderline for myocarditis, lacking definitive evidence of myocyte injury [33].

Based on the degree of $\mathrm{CD}^{+} \mathrm{T}$-cell infiltration, three of the immune checkpoint inhibitor myocarditis cases were classified as high-grade immune checkpoint inhibitor myocarditis (>50 $\mathrm{CD}^{+}$cells/hpf) and seven as low-grade immune checkpoint inhibitor myocarditis $\left(\leq 50 \mathrm{CD}^{+}\right.$cells/ hpf). The degrees of $\mathrm{CD}^{+}$and $\mathrm{CD}^{+}$T-cell infiltration in high-grade immune checkpoint inhibitor myocarditis were both similar to that in grade $2 \mathrm{R}$ acute cellular rejection, but were greater than seen in low-grade immune checkpoint inhibitor myocarditis (Figs. 1 and 2). However the degree of
Table 1 Immune checkpoint inhibitor myocarditis patient characteristics

\begin{tabular}{llll}
\hline & High grade & Low grade & $P$ \\
\hline$n$ & 3 & 7 & \\
Age (years) & $73 \pm 5$ & $71 \pm 6$ & 0.86 \\
Female sex, $n$ (\%) & $2(67)$ & $0(0)$ & 0.07 \\
Left ventricular ejection fraction (\%) & $51 \pm 8$ & $55 \pm 6$ & 0.73 \\
Serum troponin-T (ng/L) & $1430 \pm 510$ & $125 \pm 91$ & 0.12 \\
Interval first treatment to biopsy/ & $27 \pm 5$ & $307 \pm 139$ & 0.09 \\
autopsy (days) & & & \\
Cycles of immune checkpoint & $1.3 \pm 0.3$ & $6.9 \pm 3.7$ & 0.19 \\
inhibition therapy & & & \\
Cancer type, $n$ (\%) & & & 0.79 \\
Melanoma & $1(33)$ & $3(43)$ & \\
Lung & $1(33)$ & $1(14)$ & \\
Other & $1(33)$ & $3(43)$ & \\
Immune checkpoint inhibitor, $n(\%)$ & & & 0.79 \\
Nivolumab (anti-PD-1) & $1(33)$ & $3(43)$ & \\
Pembrolizumab (anti-PD-1) & $1(33)$ & $3(43)$ & \\
Ipilimumab (anti-CTLA-4) + & $1(33)$ & $1(14)$ & \\
Nivolumab (anti-PD-1) & & & \\
\hline
\end{tabular}

Values expressed as mean \pm SE or $n(\%)$

${ }^{\mathrm{a} C h o l a n g i o c a r c i n o m a, ~ s a l i v a r y ~ g l a n d ~ c a r c i n o m a, ~ o r a l ~ s q u a m o u s ~ c e l l ~}$ carcinoma, and renal cell carcinoma

macrophage infiltration in high-grade immune checkpoint inhibitor myocarditis was greater than that seen in grade $2 \mathrm{R}$ acute cellular rejection. Interestingly, the ratio of $\mathrm{CD} 68^{+}$ cells to $\mathrm{CD}^{+}$cells $(\mathrm{CD} 68 / \mathrm{CD} 3)$ was similar in low-grade immune checkpoint inhibitor myocarditis and high-grade immune checkpoint inhibitor myocarditis, but for both immune checkpoint inhibitor myocarditis groups, the CD68/ CD3 ratio was greater than that seen in grade $2 \mathrm{R}$ acute cellular rejection $(P=0.01)$, indicating immune checkpoint inhibitor myocarditis has more lymphohistiocytic character than does grade $2 \mathrm{R}$ acute cellular rejection, which manifests with a lymphocytic pattern of inflammation. There were no differences between the three groups in terms of the ratio of $\mathrm{CD}^{+}$cells to $\mathrm{CD}^{+}$cells $(\mathrm{CD} 8 / \mathrm{CD} 3)$ or the density of tryptase $^{+}$mast cells (Fig. 2). Occasional eosinophils were identified in $2(67 \%)$ of the high-grade immune checkpoint inhibitor myocarditis cases, 2 (29\%) of the low-grade immune checkpoint inhibitor myocarditis cases, and 4 ( $31 \%$ ) of the grade $2 \mathrm{R}$ acute cellular rejection cases. None of the cases contained giant cells or granulomas.

Variable numbers of macrophages were found to stain for PD-L1, and occasional myocytes were observed to stain for PD-L1 with either a diffuse cytoplasmic pattern or a diffuse membranous pattern (Fig. 3a). Variable numbers of endothelial cells were also observed to stain for PD-L1 in all three groups (not shown). Definitive lymphocyte PD-L1 staining was not observed. There was a trend toward 


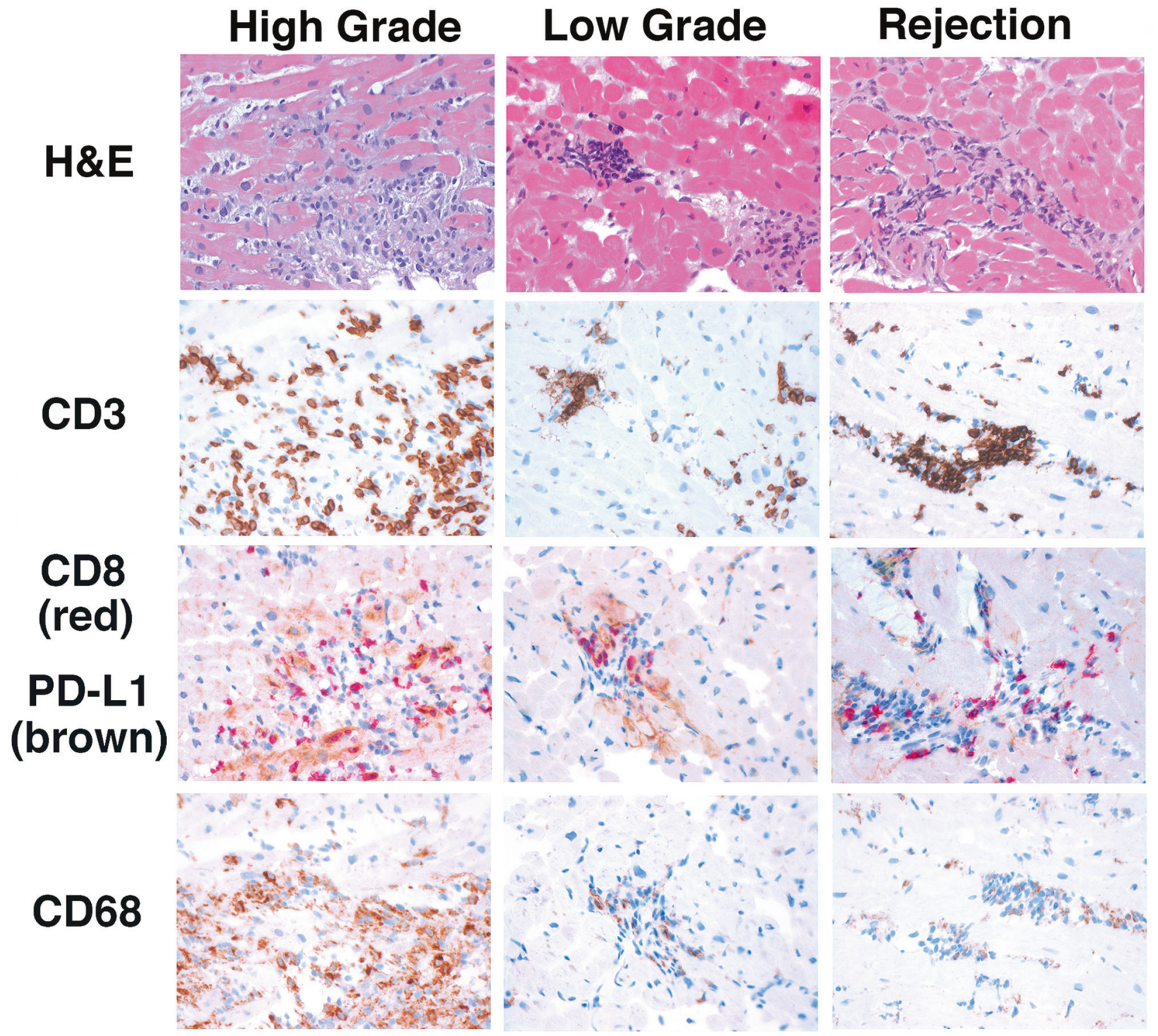

Fig. 1 Immunohistochemical staining of immune checkpoint inhibitor myocarditis and allograft rejection. Shown are representative histologic images at $\times 400$ magnification of H\&E stained slides and immunohistochemical stains for CD3, CD68, and the combination PD-L1/CD8 double stain with PD-L1 in brown and CD8 in red for a high-grade immune checkpoint inhibitor myocarditis case (high grade), a low-grade immune checkpoint inhibitor myocarditis case (low grade), and a case of grade $2 \mathrm{R}$ acute cellular rejection (Rejection)...(Color figure online) increasing densities of PD-L1 ${ }^{+}$macrophages in the immune checkpoint inhibitor myocarditis groups compared with grade $2 \mathrm{R}$ acute cellular rejection (Fig. 3b). Interestingly, the percentage of macrophages staining for PD-L1 was higher in the low-grade immune checkpoint inhibitor myocarditis cases compared with the high-grade immune checkpoint inhibitor myocarditis and grade $2 \mathrm{R}$ acute cellular rejection cases (Fig. 3c). After log transformation, the percentage of PD-L1 ${ }^{+}$macrophages was inversely correlated with the degree of $\mathrm{CD}^{+}{ }^{+}$T-cell infiltration $(P=0.03$, Fig. 3d). PD$\mathrm{L}^{+}$myocytes were present in all of the immune checkpoint inhibitor myocarditis cases but only about half of the grade $2 \mathrm{R}$ acute cellular rejection cases (Fig. 3e). Frequent PD-L1 ${ }^{+}$ myocytes ( $>10 / 10 \mathrm{hpf})$ were seen in two of the three highgrade immune checkpoint inhibitor myocarditis cases but none of the low-grade immune checkpoint inhibitor myocarditis or grade $2 \mathrm{R}$ acute cellular rejection cases (Fig. 3f). The frequency of total PD-L1 ${ }^{+}$positive cells (myocytes + macrophages) was lower in the grade $2 \mathrm{R}$ acute cellular rejection cases compared with both high-grade immune checkpoint inhibitor myocarditis $(P=0.003)$ and low-grade immune checkpoint inhibitor myocarditis $(P=0.01$, Fig. 3g). Analysis of ten endomyocardial biopsies from cardiac transplant patients without rejection as well as ten native heart biopsies/resections without myocarditis showed variable endothelial staining for PD-L1 in all cases but no 

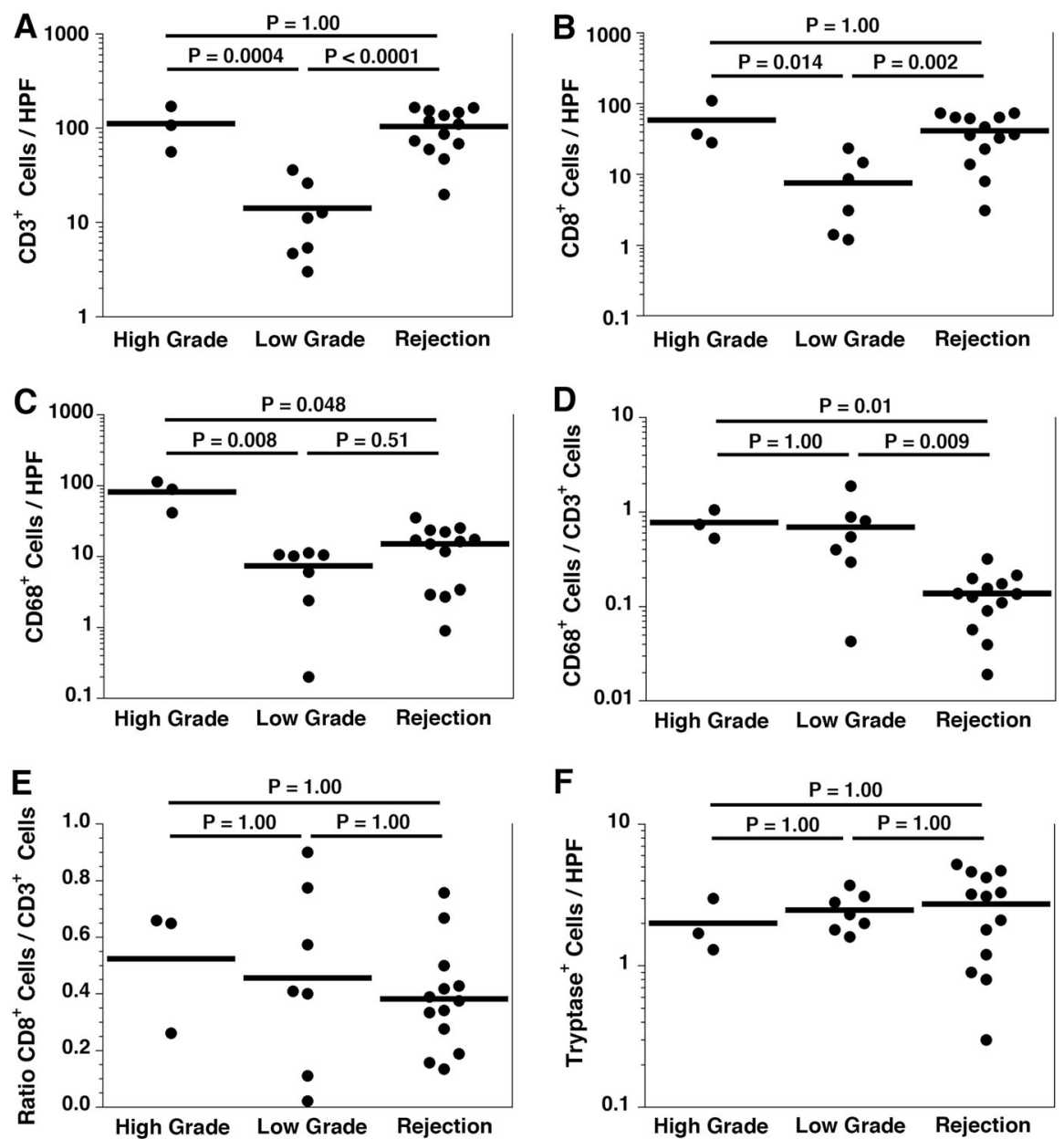

Fig. 2 Comparing the inflammatory infiltrates in high-grade immune checkpoint inhibitor myocarditis, low-grade immune checkpoint inhibitor myocarditis, and allograft rejection. Depicted are dots plots comparing high-grade immune checkpoint inhibitor myocarditis (high grade), low-grade immune checkpoint inhibitor myocarditis (low grade), and grade $2 \mathrm{R}$ acute cellular rejection (rejection) for the number of cells/hpf expressing CD3 (a), CD8 (b), CD68 (c), as well as the ratio of $\mathrm{CD} 8^{+}$cells $/ \mathrm{CD}^{+}$cells $(\mathbf{d})$, and the ratio of $\mathrm{CD} 8^{+}$cells to $\mathrm{CD}^{+}$cells $(\mathbf{e})$. There are more lymphocytes and macrophages in high-

myocyte PD-L1 staining (Fig. 3h-j). Necrotic C4D ${ }^{+}$myocytes were identified in all of the high-grade immune checkpoint inhibitor myocarditis cases, most of the lowgrade immune checkpoint inhibitor myocarditis cases, but only a small number of the grade $2 \mathrm{R}$ acute cellular rejection cases (Fig. 4). Extensive necrotic $\mathrm{C}_{4} \mathrm{D}^{+}$myocytes $(>5 / 10$ hpf) were seen in all of the high-grade immune checkpoint inhibitor myocarditis cases but none of the low-grade immune checkpoint inhibitor myocarditis cases $(P=0.008)$ and only one of the grade $2 \mathrm{R}$ acute cellular rejection cases $(P=0.007)$.

Compared with the patients with low-grade immune checkpoint inhibitor myocarditis, in the patients with highgrade immune checkpoint inhibitor myocarditis there were nonsignificant trends toward higher serum troponin levels,

grade immune checkpoint inhibitor myocarditis compared with lowgrade immune checkpoint inhibitor myocarditis. High-grade immune checkpoint inhibitor myocarditis has comparable numbers of lymphocytes to grade $2 \mathrm{R}$ acute cellular rejection, but more macrophages than are present in grade $2 \mathrm{R}$ acute cellular rejection. The CD68/CD3 ratio is higher in immune checkpoint inhibitor myocarditis than acute cellular rejection. There is no difference in the CD8/CD3 ratios or the densities of tryptase ${ }^{+}$mast cells (f)

shorter interval from first immune checkpoint inhibition treatment, and fewer cycles of immune checkpoint inhibition treatment. All of the low-grade immune checkpoint inhibitor myocarditis patients were male, but two of the three high-grade immune checkpoint inhibitor myocarditis patients were female. There were no differences between the two immune checkpoint inhibitor myocarditis groups regarding age, left ventricular ejection fraction, specific immune checkpoint inhibitor therapies, or type of cancer being treated (Table 1). All of the patients with high-grade immune checkpoint inhibitor myocarditis died. Two of the patients with high-grade immune checkpoint inhibitor myocarditis were diagnosed on endomyocardial biopsy and died 30 days later despite immunosuppressive therapy. One of these patients received high-dose corticosteroids, 

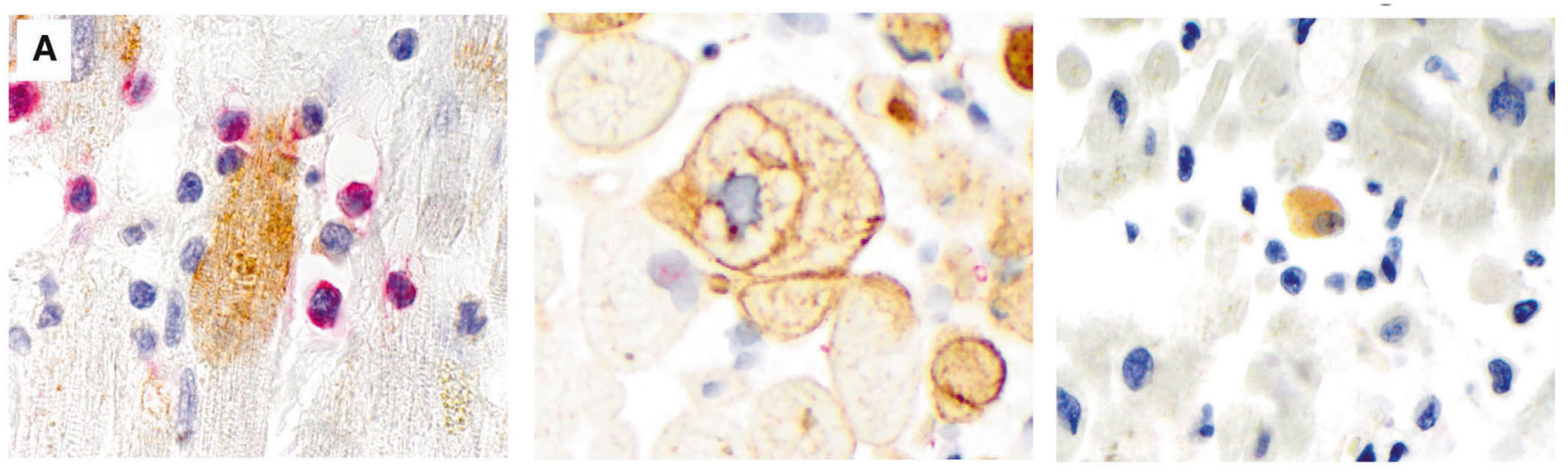

B

C

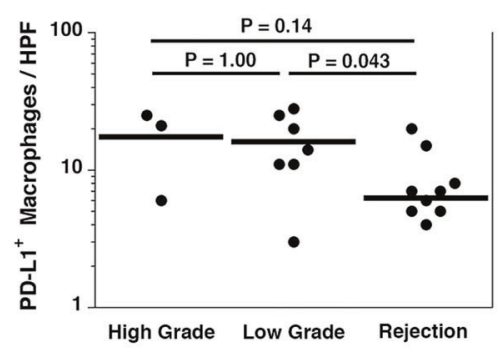

E

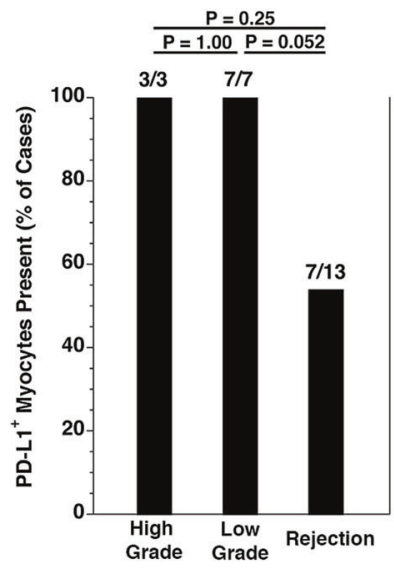

$\mathbf{F}$

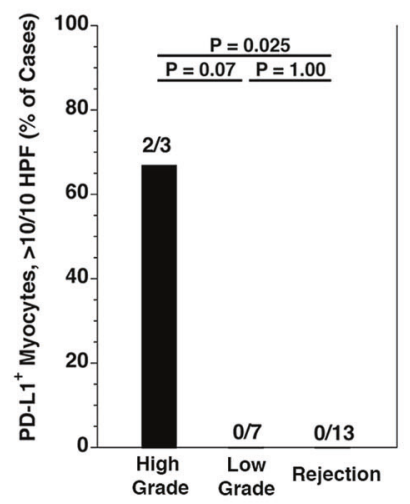

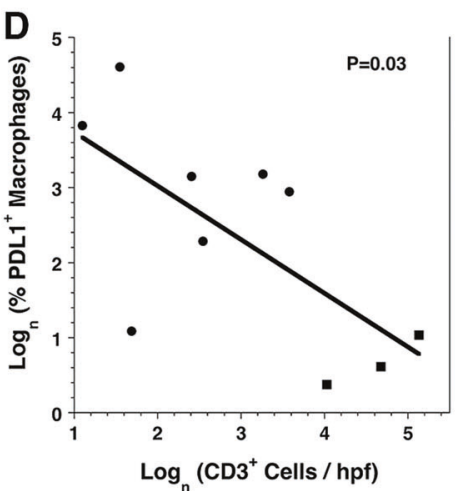

G

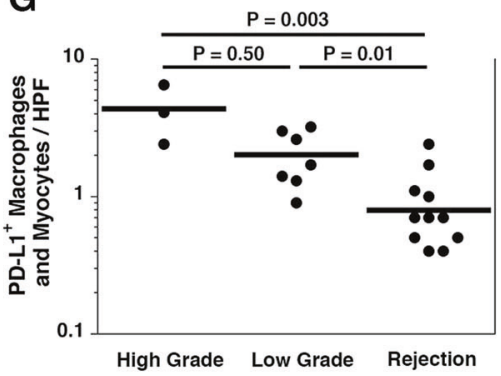

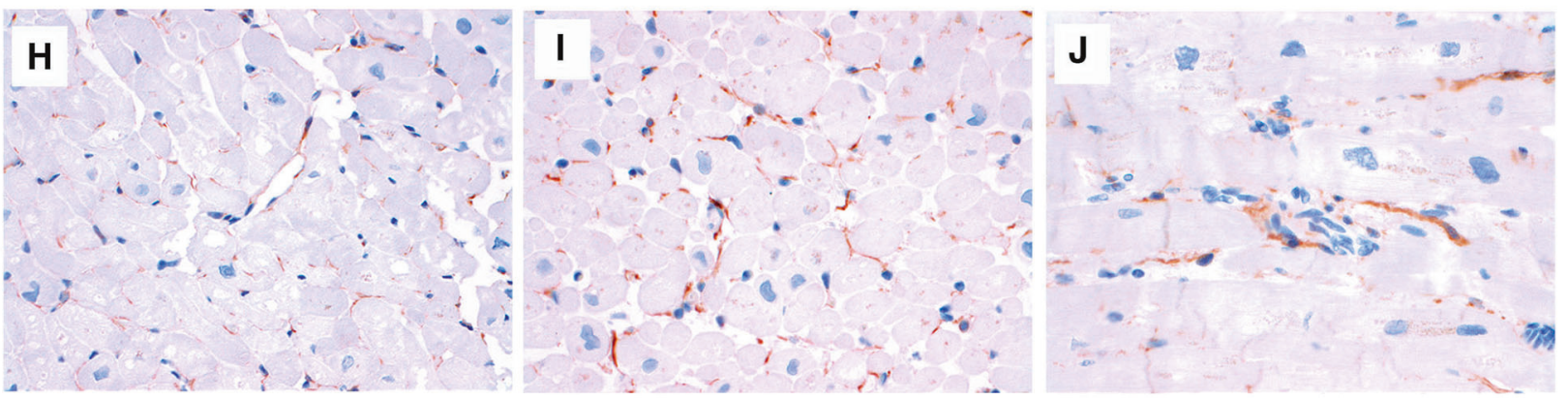

infliximab, and mycophenolate mofetil and yet showed persistent myocarditis at autopsy (not shown). The third patient with high-grade immune checkpoint inhibitor myocarditis was diagnosed at autopsy. In contrast to high-grade immune checkpoint inhibitor myocarditis, the seven patients with low-grade immune checkpoint inhibitor myocarditis were all alive on follow-up $(P=0.008)$, with a median follow-up interval of 199 days and a range of 54-335 days. Selected pathologic and clinical features, which may be useful for discriminating high-grade immune 
4 Fig. 3 PD-L1 expression by macrophages and cardiac myocytes. PD$\mathrm{L}^{+}$myocytes were identified with either a diffuse cytoplasmic staining pattern (a left) or a membranous pattern (a middle). Interstitial PD-L1 ${ }^{+}$macrophages were also identified (a right). Dot plots are shown comparing the three groups for the density of $\mathrm{PD}-\mathrm{L}^{+}$macrophages (b) and the percentage of macrophages expressing PD-L1 (c). A linear regression analysis of the ten immune checkpoint inhibitor myocarditis cases shows the relationship between the percentage of macrophages expressing PD-L1 and the density of $\mathrm{CD}^{+}$cells $(\mathbf{d})$. Shown are bar graphs depicting the percentage of cases in each group with PD-L1 ${ }^{+}$myocytes (e) and the percentage of cases with more than 10 PD-L1 ${ }^{+}$myocytes/10 hpf (f); the numbers just above each bar indicate the number of patients with the feature over the total number of patients in each group. The sum of the densities of the PD-L1 ${ }^{+}$ macrophages and the PD- $\mathrm{L1}^{+}$myocytes was greater in the immune checkpoint inhibitor myocarditis groups than in the $2 \mathrm{R}$ acute cellular rejection group (g). Immunohistochemical stains on an endomyocardial biopsy from a cardiac transplant patient without rejection (h), a native endomyocardial biopsy that was negative for myocarditis (i), and a left ventricular apical core section removed during the implantation of a left ventricular assist device in a patient with heart failure (j), all show variable endothelial PD-L1 staining, but no staining of the myocytes for PD-L1

checkpoint inhibitor myocarditis from low-grade immune checkpoint inhibitor myocarditis, are shown in Fig. 5.

\section{Discussion}

A key finding of this study is that immune checkpoint inhibitor myocarditis can be divided into high-grade and low-grade forms based on the density of the inflammatory infiltration, and that these grades of immune checkpoint inhibitor myocarditis so defined correlate with clinical outcome. High-grade immune checkpoint inhibitor myocarditis is also characterized by significantly more myocyte necrosis, and, thus, can be considered a necrotizing myocarditis analogous to acute necrotizing eosinophilic myocarditis and giant cell myocarditis $[33,36]$. Similar to those conditions, immune checkpoint inhibitor myocarditis does have substantial lymphohistiocytic character. However, while occasional eosinophils were identified in $40 \%$ of the immune checkpoint inhibitor myocarditis cases in this series, the degree of eosinophilic infiltration is much less in immune checkpoint inhibitor myocarditis than typically encountered in acute necrotizing eosinophilic myocarditis and giant cell myocarditis [36]. The histologic features of immune checkpoint inhibitor myocarditis in this series were not associated with the specific type of immune checkpoint inhibitor utilized. Importantly, in contrast to a recent study of immune checkpoint inhibitor associated hepatitis [37], immune checkpoint inhibitor myocarditis does not appear to selectively show granulomatous inflammation in the setting of CTLA-4 inhibition.

An unexpected finding of this study was that the percentage of macrophages that expressed PD-L1 was inversely related to the degree of the inflammatory infiltrate, and, thus, the grade of immune checkpoint inhibitor myocarditis. Low PD-L1 expression on macrophages has been associated with M2 polarization [38]. Thus, the lower percentage of $\mathrm{PD}-\mathrm{L}^{+}$macrophages in the high-grade immune checkpoint inhibitor myocarditis cases compared with the low-grade immune checkpoint inhibitor myocarditis cases seen here could be a result of an influx of reparative M2polarized macrophages in response to the enhanced myocyte necrosis in the high-grade immune checkpoint inhibitor myocarditis cases. Interestingly in septic patients, PD-L1 expression on monocytes was recently found to be decreased in nonsurvivors compared with survivors, similar to our observations in this series of myocarditis patients [39]. However, a previous study had found the opposite with lower monocyte PD-L1 expression in survivors compared with nonsurvivors of sepsis [40]. The role of the macrophages in immune checkpoint inhibitor myocarditis and the significance of macrophage PD-L1 expression in this disease will require further studies.

In general regardless of grade, immune checkpoint inhibitor myocarditis does appear to be distinct from typical ISHLT grade 2R cardiac allograft rejection. Primarily, immune checkpoint inhibitor myocarditis is more lymphohistiocytic than grade $2 \mathrm{R}$ cardiac allograft rejection with an increased CD68/CD3 ratio. Immune checkpoint inhibitor myocarditis is also distinguished by increased overall expression of PD-L1, which is due to expression on both macrophages and myocytes. The increased expression of PD-L1 on myocytes in high-grade immune checkpoint inhibitor myocarditis compared with grade $2 \mathrm{R}$ acute cellular rejection despite comparable degrees of lymphocytic infiltration is particularly interesting. This could simply be a result of the more necrotizing nature of high-grade immune checkpoint inhibitor myocarditis or possibly a selective upregulation of PD-L1 due to therapeutic PD-1 blockade. Given that only about half of the grade $2 \mathrm{R}$ acute cellular rejection cases showed any PD-L1 myocyte staining and none of these cases showed frequent myocyte staining of $>10 / 10 \mathrm{HPF}$, elucidation of the role of myocyte PD-L1 expression in allograft rejection and the diagnostic utility of PD-L1 staining in the setting of allograft rejection will require further studies.

It will be important to understand the relationship between PD-L1 staining of myocytes and myocyte necrosis. Within the high-grade immune checkpoint inhibitor myocarditis group, there was an average of $15 \mathrm{C}_{4} \mathrm{D}^{+}$myocytes/ HPF but only 2.6 PD-L1 ${ }^{+}$myocytes/HPF. Thus, most of the necrotic myocytes in the high-grade immune checkpoint inhibitor myocarditis group do not stain for PD-L1. In addition, many of the myocytes expressing PD-L1 do not appear overtly necrotic on the immunohistochemical stain (Fig. 3a). Comparing serial sections, some of the PD-L1 ${ }^{+}$ 
Fig. $4 \mathrm{C}^{2} \mathrm{D}^{+}$necrotic myocytes. Shown are histologic images at $\times 400$ magnification of C4D immunohistochemical stains. For high-grade immune checkpoint inhibitor myocarditis (a) there are numerous necrotic $\mathrm{C}_{4} \mathrm{D}^{+}$myocytes. For low-grade immune checkpoint inhibitor myocarditis (b), there are rare C4D ${ }^{+}$necrotic myocytes. Most cases of $2 \mathrm{R}$ acute cellular rejection showed no $\mathrm{C} 4 \mathrm{D}^{+}$ necrotic myocytes (c). Shown are bar graphs depicting the percentage of cases in each group with $\mathrm{C} 4 \mathrm{D}^{+}$necrotic myocytes (d) and the percentage of cases with more than five $\mathrm{C}_{4} \mathrm{D}^{+}$necrotic myocytes/10 hpf (e); the numbers just above each bar indicate the number of patients with the feature over the total number of patients in each group
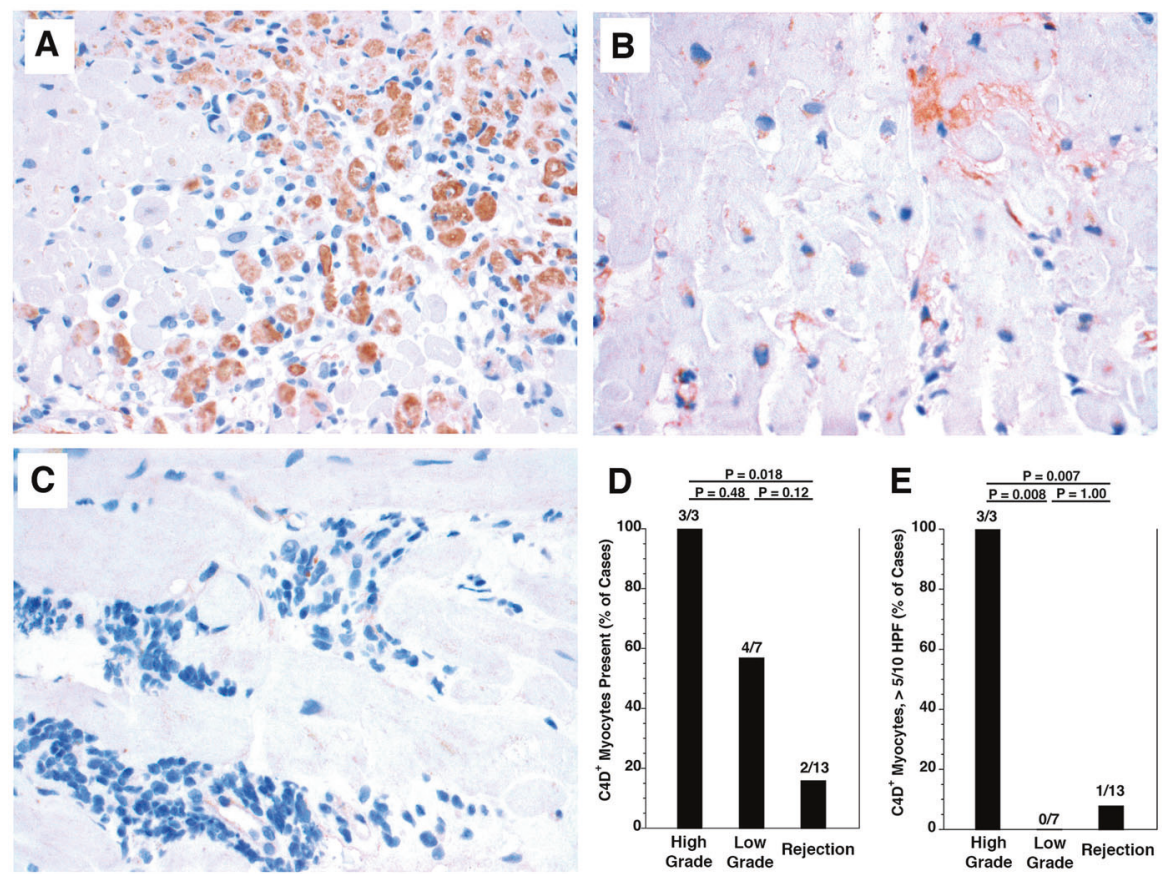

\begin{tabular}{|c|c|c|c|c|c|c|c|c|c|c|}
\hline & \multicolumn{7}{|c|}{ Low Grade } & \multicolumn{3}{|c|}{ High Grade } \\
\hline & 1 & 2 & 3 & 4 & 5 & 6 & 7 & 8 & 9 & 10 \\
\hline $\mathrm{CD}^{+}$Cells, $>50 / \mathrm{hpf}$ & & & & & & & & & & \\
\hline $\mathrm{CD}^{+}$Cells, $>25 / \mathrm{hpf}$ & & & & & & & & & & \\
\hline $\mathrm{CD} 8^{+}$Cells, $>30 / \mathrm{hpf}$ & & & & & & & & & & \\
\hline $\mathrm{C}^{2 D^{+}}$Myocytes, $>5 / 10 \mathrm{hpf}$ & & & & & & & & & & \\
\hline $\mathrm{PDL1}^{+}$Myocytes, $>10 / 10 \mathrm{hpf}$ & & & & & & & & & & \\
\hline Serum Troponin >300 ng/L & & & & & & & & & & \\
\hline Interval to Biopsy $<40$ days & & & & & & & & & & \\
\hline$<10 \%$ of Macrophages PDL1 ${ }^{+}$ & & & & & & & & & & \\
\hline$>1$ Foci of Myocyte Injury & & & & & & & & & & \\
\hline Eosinophils Present & & & & & & & & & & \\
\hline
\end{tabular}

Fig. 5 Contrasting high-grade immune checkpoint inhibitor myocarditis and low-grade immune checkpoint inhibitor myocarditis. A heat map listing selected features associated with high-grade immune checkpoint inhibitor myocarditis and indicating the presence (red box) or absence (green box) of that feature for each of the ten patients. The patients are arranged in order of increasing $\mathrm{CD}^{+}$cell density from left to right. The interval to biopsy indicates the interval from first immune checkpoint inhibition treatment to biopsy or autopsy...(Color figure online)

myocytes were in regions with $\mathrm{C} 4 \mathrm{D}^{+}$myocytes, but some were not. Four of the low-grade immune checkpoint inhibitor myocarditis cases contained PD-L1 ${ }^{+}$myocytes but not C4D ${ }^{+}$myocytes. Thus, the PD-L1 staining of the myocytes does not appear to be simply secondary to necrosis. However, some of the PD- $\mathrm{L}^{+}$myocytes in immune checkpoint inhibitor myocarditis may also be necrotic. Additional double-labeling studies will be required to address this issue more completely.
While this study indicates that there are different grades of immune checkpoint inhibitor myocarditis, precisely defining these grades in the most clinically useful manner will require larger studies. Even though CD3 was used to define the grades in this study, it is clear from Fig. 5 that CD8 and CD68 may be equally useful for distinguishing these grades. If the ISHLT grading system had been applied to these cases of immune checkpoint inhibitor myocarditis, then five of the cases would be graded as $1 R$ and five as $2 R$. Thus applying the ISHLT system and focusing on cases with a grade of $2 \mathrm{R}$ or above, would be a quick and sensitive method to identify the high-grade immune checkpoint inhibitor myocarditis pathology described here, but would not be specific, as two of the low-grade immune checkpoint inhibitor myocarditis cases would also be graded as $2 \mathrm{R}$ (Fig. 5). Likewise, the clinical features of high serum troponin $(>300 \mathrm{ng} / \mathrm{L})$ and short interval from first immune checkpoint inhibitor treatment to biopsy/autopsy ( $<40$ days) were also sensitive but not specific for high-grade immune checkpoint inhibitor myocarditis. The presence of eosinophils did trend with high-grade immune checkpoint inhibitor myocarditis compared with low-grade immune checkpoint inhibitor myocarditis, but lacked both sensitivity and specificity for differentiating the grades.

This study has several limitations. While this is the largest series of immune checkpoint inhibitor myocarditis cases yet reported, the number of patients was relatively small, particularly in the high-grade immune checkpoint inhibitor myocarditis group. This study was focused on patients who had undergone endomyocardial biopsy or autopsy and thus may contain selection bias, which may 
render the patients in this study to not be fully representative of all patients with immune checkpoint inhibitor myocarditis. All of the high-grade immune checkpoint inhibitor myocarditis patients and one of the low-grade immune checkpoint inhibitor myocarditis patients in this study had received corticosteroids prior to biopsy/autopsy, and these treatments may have altered histologic features such as the expression of PD-L1 by macrophages and the presence of eosinophils.

In conclusion, immune checkpoint inhibitor myocarditis occurs in two forms, a high-grade form with a more fulminant clinical course, and a low-grade form with a more indolent clinical course. Compared with ISHLT grade $2 \mathrm{R}$ acute cellular rejection, immune checkpoint inhibitor myocarditis is characterized by more lymphohistiocytic inflammation with an increased CD68/CD3 ratio and increased numbers of PD-L1 ${ }^{+}$macrophages and myocytes.

\section{Compliance with ethical standards}

Conflict of interest The authors declare that they have no conflict of interest.

Publisher's note Springer Nature remains neutral with regard to jurisdictional claims in published maps and institutional affiliations.

\section{References}

1. Pardoll DM. The blockade of immune checkpoints in cancer immunotherapy. Nat Rev Cancer. 2012;12:252-64.

2. Baumeister SH, Freeman GJ, Dranoff G, Sharpe AH. Coinhibitory pathways in immunotherapy for cancer. Ann Rev Immunol. 2016;34:539-73.

3. Ribas A, Wolcho JD. Cancer immunotherapy using checkpoint blockade. Science. 2018;359:1350-5.

4. Postow MA, Sidlow R, Hellmann MD. Immune-related adverse events associated with immune checkpoint blockade. N Engl J Med. 2018;378:158-68.

5. Michot JM, Bigenwald C, Champiat S, Collins M, Carbonnel F, Postel-Vinay $\mathrm{S}$, et al. Immune-related adverse events with immune checkpoint blockade: a comprehensive review. Eur J Cancer. 2016;54:139-48.

6. Bertrand A, Kostine M, Barnetche T, Truchetet ME, Schaeverbeke $\mathrm{T}$. Immune related adverse events associated with antiCTLA-4 antibodies: systematic review and meta-analysis. BMC Med. 2015;13:211.

7. Palmieri DJ, Carlino MS. Immune checkpoint inhibitor toxicity. Curr Oncol Rep. 2018;20:72.

8. Atallah-Yunes SA, Kadado AJ, Kaufman GP, HernandezMontfort J. Immune checkpoint inhibitor therapy and myocarditis: a systematic review of reported cases. J Cancer Res Clin Oncol. 2019;145:1527-57.

9. Mahmood SS, Fradley MG, Cohen JV, Nohria A, Reynolds KL, Heinzerling LM, et al. Myocarditis in patients treated with immune checkpoint inhibitors. J Am Coll Cardiol. 2018;71:1755-64.

10. Salem JE, Manouchehri A, Moey M, Lebrun-Vignes B, Bastarache L, Pariente A, et al. Cardiovascular toxicities associated with immune checkpoint inhibitors: an observational, retrospective, pharmacovigilance study. Lancet Oncol. 2018;19:1579-89.

11. Hu JR, Florido R, Lipson EJ, Naidoo J, Ardehali R, Tocchetti CG, et al. Cardiovascular toxicities associated with immune checkpoint inhibitors. Cardiovasc Res. 2019;115:854-68.

12. Raikhelkar J, Uriel N. Immune checkpoint inhibitor myocarditis. Curr Opin Cardiol. 2019;34:303-6.

13. Escudier M, Cautela J, Malissen N, Ancedy Y, Orabona M, Pinto J, et al. Clinical features, management, and outcomes of immune checkpoint inhibitor-related cardiotoxicity. Circulation. 2017;136: 2085-7.

14. Johnson DB, Balko JM, Compton ML, Chalkias S, Gorham J, Xu Y, et al. Fulminant myocarditis with combination immune checkpoint blockade. N Engl J Med. 2016;375:1749-55.

15. Chen Q, Huang DS, Zhang LW, Li YQ, Wang HW, Liu HB. Fatal myocarditis and rhabdomyolysis induced by nivolumab during the treatment of type B3 thymoma. Clin Toxicol. 2018;56:667-71.

16. Fukasawa Y, Sasaki K, Natsume M, Nakashima M, Ota S, Watanabe $\mathrm{K}$, et al. Nivolumab-induced myocarditis concomitant with myasthenia gravis. Case Rep Oncol. 2017;10:809-12.

17. Gallegos C, Rottmann D, Nguyen VQ, Baldassarre LA. Myocarditis with checkpoint inhibitor immunotherapy: case report of late gadolinium enhancement on cardiac magnetic resonance with pathology correlate. Eur Heart J Case Rep. 2019;3:yty149

18. Ganatra S, Neilan TG. Immune checkpoint inhibitor associated myocarditis. Oncologist. 2018;23:879-86.

19. Glass CK, Mitchell RN. Winning the battle, but losing the war: mechanisms and morphology of cancer-therapy-associated cardiovascular toxicity. Cardiovasc Pathol. 2017;30:55-63.

20. Heinzerling L, Ott PA, Hodi FS, Husain AN, Tajmir-Riahi A, Pauschinger M, et al. Cardiotoxicity associated with CTLA4 and PD1 blocking immunotherapy. J Immunother Cancer. 2016;4:50.

21. Imai R, Ono M, Nishimura N, Suzuki K, Komiyama N, Tamura T. Fulminant myocarditis caused by an immune checkpoint inhibitor: a case report with pathologic findings. J Thorac Oncol. 2019;14: e36-8.

22. Jain V, Mohebtash M, Rodrigo ME, Ruiz G, Atkins MB, Barac A. Autoimmune myocarditis caused by immune checkpoint inhibitors treated with antithymocyte globulin. J Immunother. 2018;41:332-5.

23. Koelzer VH, Rothschild SI, Zihler D, Wicki A, Willi B, Willi N, et al. Systemic inflammation in a melanoma patient treated with immune checkpoint inhibitors-an autopsy study. J Immunother Cancer. 2016;4:13.

24. Läubli H, Balmelli C, Bossard M, Pfister O, Glatz K, Zippelius A. Acute heart failure due to autoimmune myocarditis under pembrolizumab treatment for metastatic melanoma. J Immunother Cancer. 2015;3:11.

25. Martinez-Calle N, Rodriguez-Otero P, Villar S, Mejías L, Melero I, Prosper F, et al. Anti-PD1 associated fulminant myocarditis after a single pembrolizumab dose: the role of occult pre-existing autoimmunity. Haematologica. 2018;103:318-21.

26. Matson DR, Accola MA, Rehrauer WM, Corliss RF. Fatal myocarditis following treatment with the PD-1 inhibitor nivolumab. J Forensic Sci. 2018;63:954-7.

27. Norwood TG, Westbrook BC, Johnson DB, Litovsky SH, Terry $\mathrm{NL}, \mathrm{McKee} \mathrm{SB}$, et al. Smoldering myocarditis following immune checkpoint blockade. J Immunother Cancer. 2017;5:91.

28. Tadokoro T, Keshino E, Makiyama A, Sasaguri T, Ohshima K, Katano H, et al. Acute lymphocytic myocarditis with anti-PD-1 antibody nivolumab. Circ Heart Fail. 2016;9:e003514.

29. Tajmir-Riahi A, Bergmann T, Schmid M, Agaimy A, Schuler G, Heinzerling L. Life-threatening autoimmune cardiomyopathy reproducibly induced in a patient by checkpoint inhibitor therapy. J Immunother. 2018;41:35-8. 
30. Tay RY, Blackley E, McLean C, Moore M, Bergin P, Gill S, et al. Successful use of equine anti-thymocyte globulin (ATGAM) for fulminant myocarditis secondary to nivolumab therapy. $\mathrm{Br} \mathrm{J}$ Cancer. 2017;117:921-4.

31. Yamaguchi S, Morimoto R, Okumura T, Yamashita Y, Haga T, Kuwayama $\mathrm{T}$, et al. Late-onset fulminant myocarditis with immune checkpoint inhibitor nivolumab. Can J Cardiol. 2018;34:812.e1-3.

32. Reuben A, Petaccia de Macedo M, McQuade J, Joon A, Ren Z, Calderone $\mathrm{T}$, et al. Comparative immunologic characterization of autoimmune giant cell myocarditis with ipilimumab. Oncoimmunology. 2017;6:e1361097.

33. Leone O, Veinot JP, Angelini A, Baandrup UT, Basso C, Berry G, et al. Consensus statement on endomyocardial biopsy from the European Association for Cardiovascular Pathology and the Society for Cardiovascular Pathology 2011. Cardiovasc Pathol. 2012;21:245-74.

34. Stewart S, Winters GL, Fishbein MC, Tazelaar HD, Kobashigawa J, Abrams J, et al. Revision of the 1990 working formulation for the standardization of nomenclature in the diagnosis of heart rejection. J Heart Lung Transpl. 2005;24:1710-20.

35. Stone JR, Basso C, Baandrup UT, Bruneval P, Butany J, Gallagher PJ, et al. Recommendations for processing cardiovascular surgical pathology specimens: a consensus statement from the Standards and Definitions Committee of the Society for Cardiovascular Pathology and the Association for European Cardiovascular Pathology. Cardiovasc Pathol. 2012;21:2-16.

36. Stone JR. Diagnostic biopsies of the native heart. In: Winters GL, editor. Current concepts in cardiovascular pathology, surgical pathology clinics. Philadelphia PA: Saunders; 2012. p. 401-16.

37. de Martin E, Michot JM, Papouin B, Champiat S, Mateus C, Lambotte $\mathrm{O}$, et al. Characterization of liver injury induced by cancer immunotherapy using immune checkpoint inhibitors. J Hepatol. 2018;68:1181-90.

38. Singhal S, Stadanlick J, Annunziata MJ. Human tumor-associated monocytes/macrophages and their regulation of $\mathrm{T}$ cell responses in early-stage lung cancer. Sci Tranl Med. 2019;11:eaat1500.

39. Ferreira da Mota NV, Brunialti MKC, Santos SS, Machado FR, Assuncao M, Azevedo LCP, et al. Immunophenotyping of monocytes during human sepsis shows impairment in antigen presentation: a shift toward nonclassical differentiation and upregulation of FC-gamma-RI-receptor. Shock. 2018;50:293-300.

40. Guignant C, Lepape A, Huang X, Kherouf H, Denis L, Poitevin F, et al. Programmed death-1 levels correlate with increased mortality, nosocomial infection and immune dysfunctions in septic shock patients. Crit Care. 2011;15:R99. 